\title{
Percepção do enfermeiro sobre seu ambiente de trabalho e processo de enfrentamento à pandemia do SARS-CoV-2
}

Nurse's perception about their work environment and the process of coping with the pandemic of SARS-CoV-2

Percepción de las enfermeras sobre su entorno de trabajo y el proceso de hacer frente a la pandemia de SARS-CoV-2

Ester Coelho de Lima Fernandes ORCID: https://orcid.org/0000-0003-3040-4121 Universidade CEUMA, Brasil E-mail: estercoelhof10@gmail.com

Marluce Sampaio Nobre Barbosa ORCID: https://orcid.org/0000-0001-7276-6521 Universidade CEUMA, Brasil E-mail:marluce.nobre@ceuma.br

Suelane Coelho de Lima ORCID: https://orcid.org/0000-0002-4547-6817 Universidade CEUMA, Brasil E-mail: coelhosuelane80@gmail.com

Iracema Sousa Santos Mourão ORCID: https://orcid.org/0000-0003-2990-2684 Universidade Estadual do Maranhão, Brasil E-mail: iracemasts@ hotmail.com

Gabriela Caroline Silva Queiroz ORCID: https://orcid.org/0000-0003-4115-9176 Universidade CEUMA, Brasil E-mail: gabqrzz@gmail.com

Felype Hanns Alves Medeiros ORCID: https://orcid.org/0000-0001-5026-1995 Universidade Estadual do Pará, Brasil E-mail: felypehanns@ @otmail.com

Patrícia dos Santos Silva Queiroz ORCID: https://orcid.org/0000-0002-9587-1786 Universidade CEUMA, Brasil E-mail: patriciasqueiroz@gmail.com

\begin{abstract}
Resumo
Objetivo: Avaliar como o enfermeiro percebeu o seu ambiente de trabalho durante a pandemia. Método: Trata-se de uma pesquisa de natureza descritiva e de abordagem qualitativa, por analise de discurso através de questionário enviado de forma online enviado por aplicativo de conversas. A coleta de dados ocorreu de agosto a setembro de 2021. Resultados: Observou-se que mesmo com treinamentos/capacitações, os enfermeiros relacionam o medo, o estresse e outros, como fatores primórdios de adoecimento mental, percebendo ainda como os fatores de aumento do fluxo de pacientes elevem a carga de estresse dentro e fora do trabalho, levando a sofrimento mental. Conclusão: Considerando o profissional enfermeiro, ressalta-se a relevância de abordar o tema para demais profissionais e estudantes da área. Esperasse com os resultados contribuir para a reflexão do papel do enfermeiro e a carga emocional e física que estes profissionais carregam.
\end{abstract}

Palavras-chave: Saúde mental; Infecção pelo SARS-CoV-2; Covid-19; Enfermagem; Estresse; Ansiedade.

\begin{abstract}
Objective: To evaluate how nurses perceived their work environment during the pandemic. Method: This is a descriptive and qualitative research, by discourse analysis through a questionnaire sent online sent by conversation application. Data were collected from August to September 2021. Results: It was observed that even with training/training, nurses relate fear, stress and others, as early factors of mental illness, also realizing how the factors of increased flow of patients increase the burden of stress inside and outside work, leading to mental suffering. Conclusion: Considering the nursing professional, the relevance of addressing the theme to other professionals and
\end{abstract}


students in the area is emphasized. It was expected with the results to contribute to the reflection of the role of nurses and the emotional and physical load that these professionals carry.

Keywords: Mental health; SARS-CoV-2 Infection; Covid-19; Nursing; Stress; Anxiety.

\section{Resumen}

Objetivo: Evaluar cómo las enfermeras percibieron su ambiente de trabajo durante la pandemia. Método: Se trata de una investigación descriptiva y cualitativa, mediante análisis del discurso a través de un cuestionario enviado en línea enviado por la aplicación de conversación. los datos fueron recolectados de agosto a septiembre de 2021. Resultados: se observó que incluso con la capacitación / capacitación, las enfermeras relacionan el miedo, el estrés y otros, como factores tempranos de enfermedad mental, dándose cuenta también de cómo los factores de aumento del flujo de pacientes aumentan la carga de estrés dentro y fuera del trabajo, lo que lleva al sufrimiento mental. Conclusión: Considerando al profesional de enfermería, se enfatiza la relevancia de abordar el tema a otros profesionales y estudiantes del área. Se esperaba con los resultados contribuir a la reflexión del papel de las enfermeras y la carga emocional y física que llevan estos profesionales.

Palabras clave: Salud mental; Infección por el SARS-CoV-2; Covid-19; Enfermería; Estrés; Ansiedad.

\section{Introdução}

Em novembro de 2019 um surto de doença respiratória, causado pelo novo coronavírus (SARS-CoV-2), foi detectado na cidade de Wuhan, na China. Em dois meses foram confirmados milhares de casos de Covid-19 (atual denominação da doença), que resultaram em inúmeros óbitos. Em março de 2020, o novo coronavírus disseminou-se para mais de uma centena de países, continuando a causar doença respiratória e óbitos, especialmente em grupos de risco como idosos, gestantes, imunodeprimidos e outros. (Ministério da saúde, SBI, 2020)

De acordo com o Protocolo de Manejo Clínico do Coronavírus, o vírus foi denominado coronavírus da síndrome respiratória aguda grave (SARS-CoV-2) e produz a doença conhecida como COVID-19. Os sintomas com maior frequência são febre, tosse seca, cansaço, coriza, obstrução nasal, dor de garganta e diarreia, sendo que $14 \%$ dos pacientes apresentam sintomas severos (dificuldade em respirar e falta de ar), necessitando de internação para oxigenoterapia e 5\% apresentam sintomas críticos (insuficiência respiratória, risco de morte, entre outros).

Em virtude do rápido crescimento do número de profissionais infectados e mortos, o estresse, o bem-estar físico e mental tem sido alvo de diversas discussões e estudos pelos profissionais e instituições. Estudos têm sugerido que o medo de ser infectado por um vírus potencialmente fatal e de rápida disseminação, cujas origens, natureza e curso ainda são pouco conhecidos, acaba por afetar o bem-estar psicológico de muitas pessoas. (Asmundson \& Taylor, 2020; Malta et al., 2020) Sintomas de depressão, ansiedade e estresse diante da pandemia têm sido identificados na população geral (Wang et al., 2020) e, em particular, nos profissionais da saúde. (Zhang et al., in press, 2020).

Algumas questões têm permeado debates no contexto da saúde mental e o processo de trabalho dos profissionais de saúde no Brasil e no mundo.

- Qual a maior dificuldade ao tratar pacientes com sars-cov-2?

- Como você está lidando com essa nova situação?

- Você se isolou, ou está isolado da sua família?

- A instituição que você trabalha oferece apoio psicológico?

- Você apresentou sintomas de ansiedade?

Uma vez que os impactos do sars-cov-2 não se limitam apenas à danos físicos, os sintomas de ansiedade, depressão, angústia, ou transtorno do pânico (TP), variam de acordo com os profissionais enfermeiros. Alguns dos sintomas dependem da exposição desses profissionais às pessoas infectadas ou suspeitas, há indícios de que um grupo de pessoas sejam mais afetadas 
de que outros, como aqueles que trabalham diretamente com casos de sars-cov-2 em mais de um hospital, e que foram infectados durante o trabalho ou fora dele.

O isolamento social, e/ou distanciamento social é outra grande preocupação, o fator de não poder sair de casa, principalmente no período em que o paciente está notificado por suspeita de COVID-19, por um período de quatorze dias, isolados dos familiares, ou sem ter como está isolado deles, e ocasionalmente o excesso de informações e discordâncias sobre o tema, tornam favorável o aparecimento de sintomas depressivos e ansiosos.

Nessa perspectiva, pode-se afirmar que juntamente com a pandemia de COVID-19 surge um estado de pânico social em nível global e a sensação do isolamento social desencadeia os sentimentos (angústia, insegurança e medo), que podem se estender até mesmo após o controle do vírus. (Hossain et al., Pereira et al., 2020)

Naturalmente, os enfermeiros não estão fora desta contagem, trabalhar durante longos períodos em contato direto à pacientes positivos, intensifica o medo e o estresse, além de uma possível piora em profissionais com transtornos préexistentes. Paralelamente, Stroebe, et al. (2007) afirmam que não poder dar apoio e estar com os familiares, independente da gravidade do seu estado de saúde, pode se tornar um gatilho para o surgimento do sentimento de culpa e tristeza.

O fator primordial está relacionado a demanda de serviços, estes enfermeiros enfrentam a pandemia de perto, situações de perda, morte, dor e sofrimento, somados as condições de trabalho e remuneração críticas. Todos esses fatores determinam a carga de estresse e desgaste físico e psíquico.

\section{Metodologia}

\subsection{Aspectos éticos}

A pesquisa iniciou-se após aprovação do Comitê de Ética em Pesquisa, respeitadas às normas de pesquisa envolvendo seres humanos, Resolução n 466/12 e a Resolução n 510/16 do Conselho Nacional de Saúde, e na Plataforma Brasil, sob o parecer $n^{\circ} 4.748 .612$.

\subsection{Desenho, local de estudo e período.}

Trata-se de uma pesquisa de natureza descritiva de abordagem qualitativa, por analise de discurso através de questionário enviado de forma online enviado por aplicativo de conversas à coordenação do hospital de uma cidade no interior do Maranhão.

De um total de 20 enfermeiros 19 aceitaram e foram participantes desse estudo. Após concordar e estar ciente do Termo de Consentimento Livre e Esclarecido, entre agosto e setembro de 2021, realizam-se entrevistas com questões objetivas e subjetivas, está por último com o objetivo de o respondente dê sua opinião sem induzir sua resposta com opções préestabelecidas pelo autor, permitindo que haja resultados mais profundos e precisos acerca do tema trabalhado.

\subsection{Coleta, organização de dados e análise de dados.}

$\mathrm{O}$ estudo sendo realizado com a ferramenta Google Forms em plataforma online e garante o anonimato dos entrevistados, e serão representados pela letra $\mathrm{E}$, seguida de números.

Os dados coletados com a ferramenta Google Forms são automaticamente analisados e transformados em relatório, sendo tabulados pela própria plataforma. Ressalva-se que o relatório final é submetido e avaliado pelos autores. 


\section{Resultados e Discussão}

\subsection{Capacitação e treinamento durante a pandemia}

Considerando a importância de capacitação e treinamento, o combate à pandemia somados esforços das autoridades públicas o COFEN na resolução 636/2020 recomenda aos profissionais de enfermagem realizarem capacitação e/ou treinamento de combate e prevenção à pandemia de COVID-19. (Stroebe, 2007, Confen 2020)

Considerando ainda a importância da capacitação/treinamento dos profissionais, visto que o mesmo possibilita aos profissionais atuarem na linha de frente possibilitando a melhor assistência a paciente critico, esses profissionais devem ter a capacidade de entender os mecanismos de transmissão, prevenção e controle, além de possíveis redução de riscos de exposição e morte. No gráfico abaixo, os profissionais relatam sobre as capacitações e treinamento recebidos durante a pandemia.

Os profissionais enfermeiros afirmam terem participado de treinamento em prevenção e controle à covid-19 78,90\%, e após o treinamento $63,20 \%$ afirmam mais confiança em realizar a assistência necessária ao paciente, 42,10\% receberam um segundo treinamento e $89,50 \%$ afirmam ainda que menos de um treinamento não foram o suficiente. Porém ao longo do tempo, visto que a pandemia durou um período maior que um ano, todos sentem-se mais confiantes para dar maior assistência a pacientes infectados com covid-19.

\subsection{Percepção do enfermeiro relacionado ao ambiente hospitalar durante a pandemia}

Durante este cenário de pandemia e alta disseminação e morte, os enfermeiros foram diretamente atingidos com a mudança de fluxo de pessoas, e aumento da carga e estresse no contexto do trabalho, ainda há aqueles que trabalham em vários locais para complementar a renda, uma vez que não são bem remunerados, características determinantes para o adoecimento mental destes profissionais. 63,20\% afirmam possuir apenas um emprego, 31,60\% dois empregos, 78,90\% trabalham apenas em um local com setor covid-19 e $21,10 \%$ em dois ou mais.

A sobre carga de trabalha e a desproporção dos números de profissionais atuantes nos setores é um fator de risco, inclusive para o aumento de incidência de infecções hospitalares. O estudo ressalta ainda a acumulação de empregos e a alta rotatividade em frente a baixa remuneração, ou às condições de trabalho impostas na instituição e o elevado nível de estresse neste ambiente. Para favorecer maior satisfação no contexto do trabalho de enfermagem é necessário às organizações que promovam número adequado de profissionais, redução de carga horaria, bem como condições de serviços e remunerações adequadas. (Novaretti, et al 2014).

Gráfico 1 - apresenta a percepção quanto a maior mudança percebida pelo enfermeiro (a) no setor.

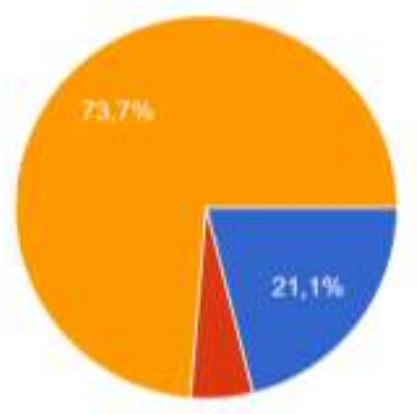

Aumento do fluxo de pessoas

Aumento da carga horaria

Aumento de estresse nos setor entre os funcionários

Fonte: Autores. 
Quando se trata da satisfação e comprometimento no trabalho, fatores esses, primordiais para uma boa convivência e melhor assistência para os pacientes, 94,70\% afirmam se sentirem satisfeitos em trabalhar no setor que estão, e 57,90\% afirmaram que o local de descanso é apropriado. E todos consideram que estar satisfeito no serviço agrega comprometimento maior entre os profissionais.

Desta forma, o lazer enquanto promoção de saúde integral, tem três funções primordiais, são elas: a de descanso, divertimento e de desenvolvimento da personalidade. O descanso é primordial para equipe, pois promove a recuperação e alívio de fatores estressantes, o lazer ou distração/divertimento são relevantes nos momentos livres de trabalho, dentro ou fora da unidade, o desenvolvimento de atividades de relaxamento forma meios de recuperação de cansaço físico, emocional e social, podendo ser atividades até mesmo de educação, reeducação, e comunicação entre equipe (o que pode promover desenvolvimento de benefícios na relação profissional). (Dumazedier J, 1973).

O descanso é primordial para equipe, 52,6\% dos enfermeiros não conseguem descansar e/ou relaxar no local de trabalho, seja por falta tempo durante o plantão ou mesmo pelo estresse ou preocupação.

A insuficiência de profissionais de enfermagem foi um fator importante para a ocorrência de infecções hospitalares \{...\} Seguindo a premissa que, ao não descansar o suficiente, mantendo-se com níveis séricos de estresse, ansiedade entre outros sintomas, o enfermeiro colabora, mesmo sem intenção, ao comprometimento da sua segurança e do paciente, a sobrecarga de trabalho torna-se um obstáculo em contrapartida das ações de educação continuada para prevenção contra a infecção hospitalar, implicando ainda na participação de treinamentos, tornando a eficácia prejudicada. (Panunto M, R, et al 2013)

\subsection{Percepção do enfermeiro relacionado a pandemia}

$\mathrm{O}$ enfermeiro trabalha em um ambiente onde as forças de vida e morte, humano e tecnológico encontram-se em luta constante, apesar de existir a equipe multidisciplinar, os enfermeiros são os principais responsável pelo acompanhamento constante aos pacientes, consequentemente possui o compromisso dentre outros de manter a homeostasia do paciente e o bom funcionamento da unidade. Ao enfermeiro compete cuidar do indivíduo nas diferentes situações, de forma integrada e contínua com os membros da equipe de saúde, para isso o enfermeiro precisa pensar criticamente, e analisar os problemas e encontrar soluções para os mesmos, assegurando sempre sua prática dentro dos princípios éticos e bioéticas da profissão. (Vargas D, 2006)

Um estudo com mapeamento mostra o índice de risco que os trabalhadores brasileiros têm de serem contaminados pelo COVID-19 durante suas atividades profissionais e os trabalhadores da saúde apresentaram de 97 a 100\% de risco de contágio desde técnicos de saúde bucal a técnicos de enfermagem, enfermeiros e médicos. (Barroso B, 2020).

O quantitativo de enfermeiros infectados com o vírus, indica que dos entrevistados, $63,2 \%$ foram infectados e 31,6\% não foram infectados e ainda 5,3\% não sabe se foi infectado e também não fez o teste, mesmo sendo disponibilizado pelo hospital.

Na Tabela 1 logo abaixo, é possível identificar que mesmo utilizando proteções enfermeiros não se sentiam seguros. 78,90\% receberam EPI suficiente para proteção, os mesmos 78,90\% disseram não serem eficazes, e outros 63,20\% afirmaram sentir inseguros mesmo utilizando os EPI. 
Tabela 1 - Percepção relacionada a proteção.

\begin{tabular}{|l|l|l|}
\hline & SIM & NÃO \\
\hline Você recebe EPI's o suficiente para sua proteção? & $78,90 \%$ & $21,10 \%$ \\
\hline Os EPI's estão sendo eficazes? & $78,90 \%$ & $21,10 \%$ \\
\hline Você sente-se INSEGURO mesmo utilizando EPI's? & $63,20 \%$ & $36,80 \%$ \\
\hline
\end{tabular}

Fonte: Autores, Google Forms (2021).

Um dos tópicos discutidos entre profissionais da área é que possuem muitas responsabilidades, além da sobrecarga de trabalho têm-se pacientes vulneráveis à diversas complicações; diversas opiniões a respeito de qual tratamento correto, tomam decisões baseada em pouquíssimos estudos; Além profissionais sem experiência, tendo formação antecipada e devem ter tomada de decisão rápida e de alto risco; Utilizam medicamentos e procedimentos de alta complexidade podendo haver riscos de erros; ser bombardeado com diversas notícias do tema diariamente, muitas delas chamadas "fake news"; taxa alta de infecção e morte entre profissionais da área; rodizio de Ministros da Saúde, e por fim, enfrentar uma pandemia sem um Ministro de Saúde. E apesar das complicações, vulnerabilidades, e estresses neste ambiente, mesmo a maioria sendo treinadas para assumir as responsabilidades, há também outros fatores influenciadores como falta de estrutura hospitalar adequada, falta de EPI's, salário sem nenhuma perspectiva de aumento, e serem contaminados, provocam esgotamentos e traumas psicológicos que possivelmente interfere na qualidade de assistência prestada. $\mathrm{O}$ vírus sendo disseminado entre profissionais colocam em riscos os familiares e obriga os profissionais a estarem isolados em um cômodo da casa. (Wang, C et al, 2020, Zhang, C. et al, 2020)

3.3.1 Os enfermeiros relataram ainda as maiores dificuldades encontradas ao lidar com quem testou positivo para o novo coronavírus:

“Aceitação da doença”. (E1).

“Resistência dos pacientes em aceitar fazer o tratamento”. (E4).

“O psicológico do paciente”. (E7)

"O nível de agitação, ansiedade que a pessoa fica! Acaba sentindo maior desconforto respiratório e apresentando piora do quadro devido ansiedade”. (E19)

“Morte”. (E9)

"A maior dificuldade está em manter o paciente isolado (distanciamento social)! Muitos não respeitam as recomendações do Ministério da Saúde! Com isso trazendo maiores riscos de transmissão!" (E2)

"Não ter tratamento adequado e ter que transferir pra outro hospital”. (E15)

"Nos casos graves quando os protocolos utilizados não surgem mais efeito, ter que encaminhar pra os hospitais de campanha às pressas para não evoluir ao óbito”. (E11)

Transtornos psíquicos imediatos são medidos pelo pico de estresse e ansiedade ou angustia, porém o luto patológico, depressão, manifestação de sintomas de estresse pós-traumático acarreta padrões de sofrimento prolongado. Padrões estes se não tratados desencadeiam patologia. (Fiocruz, 2020, Gagliato M, 2020)

\subsubsection{A percepção do enfermeiro relacionado ao maior risco de contaminação de COVID-19:}

"Falta de cuidados básicos" (E18)

“Não vacinação e não uso de mascaras mesmo vacinado”. (E16) 
"A falta de responsabilidade das pessoas umas com as outras, desrespeitando as medidas sanitárias". (E12)

"O descuido das pessoas" (E3)

"O risco de uma evolução rápida para um caso mais grave”. (E6)

"A ignorância e o comportamento torpe do ser humano". (E10)

"As aglomerações entre os amigos". (E13)

"A falta de EPI". (E5)

No Brasil, o país tem passado por dificuldade extra relacionada aos cuidados de higiene e distanciamentos socias. É importante que todos os passos essenciais para prevenção a ser fornecido à toda população exposta ao contágio para que ela possa ter a certeza de que está executando o necessário para evitar o contágio. A compreensão positiva acerca das medidas de prevenção e contenção à proliferação, é um fator que desencadeia o alivio de pressão psicológica, já que os enfermeiros consideram que não estar seguindo as medidas sanitárias necessárias para evitar o contagio aumenta o risco de morte, exposição, evolução rápida para casos graves entre outros.

\subsection{A saúde mental dos enfermeiros durante a pandemia}

A angustia é um dos sintomas mais relatados pelos enfermeiros, 31,6\% relatam quase sempre sentirem angustiados, neste cenário, o medo e a angustia intensifica sintomas de ansiedade e estresse em pessoas saudáveis e eleva sintomas daquelas com transtornos pré-existentes. 10,5\% afirmam já terem diagnostico de Transtorno do Pânico (TP), ansiedade ou depressão e após a pandemia 36,8\% afirmaram ter recebido pelo menos um diagnóstico dos citados acima.

Seguindo a mesma linha, o fator de não poder dar apoio necessário tanto a paciente quanto a familiares e ainda estar com os familiares, independente da gravidade do seu estado de saúde, poderá vir a torna-se gatilho para o surgimento do sentimento de culpa e tristeza ou angustia. (Dumazedier J, 1973)

Notou-se que 38,60\% afirmam que as instituições empregatícias disponibilizam psicólogos caso precisarem, 26\% afirmaram não precisar de apoio psicológico.

Em contra partida destes dados, 73,7\% afirmaram terem ataques de pânico, ansiedade, ou medo, em algum momento durante a pandemia. 73,7\% sentiram somente sintomas de ansiedade e outros 78,9\% sentiu-se angustiado durante a pandemia.

Em qualquer epidemia, é comum aos indivíduos sentirem-se estressados e preocupados, o medo de adoecer, de perder de subsistência, não poder trabalhar durante isolamento, e ser demitido do trabalho estão entre os principais motivos de angustia. Quando uma população é exposta a algum tipo de epidemia, poderá vir a sofrer alguma manifestação psicopatológica, caso não seja feita nenhuma intervenção para as reações e sintomas apresentados. Os fatores que desencadeiam o impacto psicossocial são relacionados a proporção da epidemia e o grau de vulnerabilidade em que a pessoa se encontra no momento. (Barroso B, 2020, Fiocruz, FOC, 2020)

Além do mais, trabalhadores da linha de frente, como enfermeiros, médicos, técnicos de enfermagem, motoristas, maqueiros e outros, sofrem estresses adicionais, a tensão utilizando equipamentos, o distanciamento mantido, mesmo sendo necessária assistência ao paciente, tornar difícil oferecer conforto ao paciente, a longa exposição e jornada longa e cansativa atrai esses fatores adicionais. 72,2\% dos enfermeiros da linha de frente afirmaram não receber o apoio psicológico adequado.

As estratégias de cuidado psíquico em situações como de pandemia recomendam acolher e procurar pessoa de confiança para conversar, e buscar profissionais quando as mesmas não forem suficientes. 


\subsection{Estratégias de enfrentamento e cuidado a saúde mental}

No decorrer dos últimos meses, inevitavelmente a vida dos trabalhadores de saúde foram fortemente afetadas. Considerando o imediatismo em cuidar da saúde mental, as instituições ofereceram tratamento psicológico mediado pelo município para trabalhadores da saúde que atuaram e ainda atuam nos centros de referência a covid no município.

A pandemia tem levado as discussões sobre saúde mental a pauta sobretudo relacionando o isolamento social à saúde física e os impactos que geram na economia que também leva a problemas relacionado a saúde mental. (Pereira MD, 2020)

Após as evidências científicas demostrarem que a equipe de saúde tem passado por sofrimento psicológico com a pandemia do COVID-19, percebeu-se a importância de tratamentos psicológicos ou psiquiátricos à essa população, uma vez que, o cuidado em saúde mental favorece a atuação do profissional no seu local de trabalho, e a ausência disso reduzirá o seu potencial de cuidado, aumentarão as chances de afastamentos, disseminações, mortes e consequências posteriores a crise desta pandemia.( LAI J, et al, 2020).

Desta forma, pensar em estratégias de enfretamento e cuidado é primordial, as intervenções incluem dimensões sociais como a exemplo o apoio social, familiar, acompanhamento através de psicoterapias online e teleconsultas, oferecendo qualquer meio em que seja possível e adequado conforme a necessidade pertinente do paciente.

Pensando nas medidas necessárias, as intervenções devem ser desenvolvidas no curto prazo principalmente o uso de primeiros socorros psicológicos com os quais promove uma relação de respeito e apoio entre os pacientes e o terapeuta/psicólogo, onde será avaliado as necessidades críticas e intervir precocemente levando a uma estabilização de sentimentos decorrentes das reações de estresse, ansiedade ou luto. A partir disso, encorajar e apoiar pensamentos positivos acerca do futuro, sem falsas garantias ou esperanças que podem piorar a situação clinica dos mesmos, além de educar os pacientes com técnicas que diminuíam os níveis de estresse e hiperatividade. (Pereira MD, 2020, Cofen 2020)

Ademais as medidas que podem ser implementadas a longo prazo, há o uso de terapias com foco no trauma vivenciado, como a terapia cognitiva-comportamental que visa desestruturar pensamentos catastróficos, negativos sobre o próprio futuro ou de terceiros. Além disso é necessário ter uma abordagem baseadas nas intervenções voltadas para a recuperação da consciência positiva, satisfatória, e na aceitação do ente perdido, caso for. Existem diversos tipos de terapias que podem ser implementadas, sendo baseadas nos tipos de necessidades dos pacientes, inclusive aquelas que podem ser complementadas com tratamento psicofarmacológico. (Ramírez-Ortiz J, 2020, Xiang YT, 2020)

\section{Considerações Finais}

No presente estudo, foram fomentados conhecimentos sobre as consequências do aumento do fluxo de pessoas nos hospitais, bem como, a perspectiva do enfermeiro relacionada a seu treinamento e capacitação para prevenção de covid-19, os impactos significativos em sua saúde mental, os sintomas psicológicos ressaltados por este estudo foram principalmente, o estresse, medo, angustia e ansiedade, que geram sofrimento psíquico e podem ocasionar o surgimento de transtornos de pânico, transtornos de ansiedade, transtorno de estresse pós-traumático (TEPT) e depressão. Ainda, medidas de estratégias direcionadas para o tratamento seja ele terapêutico ou psicofarmacológico.

Nota-se que os profissionais enfermeiros são habilitados para trabalharem no setor, no entanto, esses indivíduos devem receber atenção e cuidado psicossocial, pois a maioria se encontra em situação de vulnerabilidade, no qual os sintomas podem ser aumentados pelo meio em que trabalham.

A importância deste assunto transmite a possibilidade de reflexão sobre a importância do papel do enfermeiro e o processo de enfrentamento de situações que podem torna-se cotidianas, além do mais, buscar ajuda profissional, caso, sinta-se debilitado fisicamente e emocionalmente, assim, promovendo uma melhor perspectiva de vida pessoal e profissional. 
Portanto, faz-se necessário o ampliamento de estudos e área da pesquisa e relatos descritos neste estudo, além de diversificar metodologias de abordagem do assunto, afim de obter novas estratégias de tratamento seja psicofarmacológico ou de terapêutica seja ela em grupo, ou individual, ajustadas de acordo com a realidade atual.

\section{Agradecimentos}

Um agradecimento a todos que contribuíram para este artigo ser produzido.

\section{Referências}

Asmundson, G. J. G., \& Taylor, S. (2020). Coronaphobia: Fear and the 2019-nCoV outbreak. Journal of Anxiety Disorders, 70, 102196. https://doi.org/10.1016/j.janxdis.2020.102196

Barroso, B. I. D. L., Souza, M. B. C. A. D., Bregalda, M. M., Lancman, S., \& Costa, V. B. B. D. (2020). A saúde do trabalhador em tempos de COVID-19: reflexões sobre saúde, segurança e terapia ocupacional. Cadernos Brasileiros de Terapia Ocupacional, 28, 1093-1102.

Brasil. 2020. In: Ministério da Saúde / SAPS - Protocolo de manejo clínico do coronavírus (COVID-19) na atenção primária à saúde. Brasília - DF: Coronavírus COVID-19.

COFEN. (2020). O brasil conta comigo profissionais. Manual cadastro E capacitação. Resolução 636.2020. PORTARIA Nº 639, DE 31 DE MARÇO DE 2020 Dumazedier, J. (2004). Lazer e cultura popular. In Lazer e cultura popular (pp. 333-333).

FIOCRUZ, Fundação Oswaldo Cruz. (2020a). Cartilha Saúde Mental e Atenção Psicossocial -Informações Gerais. https://portal.fiocruz.br/documento/cartilhasaude-mental-e-atencao-psicossocial-na-pandemia-covid-19.

Gagliato, M. (2020). Guia Preliminar como lidar com os aspectos Psicossociais e de Saúde mental referente ao surto de COVID - 19. Versão 1.5 https://interagencystandingcommittee.org/system/files/2020-03/IASC Interim Briefing Note on COVID-19 Outbreak Readiness and Response Operations MHPSS\%28Portuguese\%29.pdf

Hossain, M. M., Sultana, A., \& Purohit, N. (2020). Mental health outcomes of quarantine and isolation for infection prevention: a systematic umbrella review of the global evidence. Epidemiology and health, 42. doi: https://doi.org/10.31234/OSF.IO/DZ5V2

Lai, J., Ma, S., Wang, Y., Cai, Z., Hu, J., Wei, N., \& Hu, S. (2020). Factors associated with mental health outcomes among health care workers exposed to coronavirus disease 2019. JAMA network open, 3(3), e203976-e203976.

Malta, D. C., Szwarcwald, C. L., Barros, M. B. D. A., Gomes, C. S., Machado, Í. E., Souza, P. R. B. D., \& Gracie, R. (2020). A pandemia da COVID-19 e as mudanças no estilo de vida dos brasileiros adultos: um estudo transversal, 2020. Epidemiologia e Serviços de Saúde, 29. https://doi.org/10.1590/s167949742020000400026

Novaretti, M. C. Z., Santos, E. D. V., Quitério, L. M., \& Daud-Gallotti, R. M. (2014). Sobrecarga de trabalho da Enfermagem e incidentes e eventos adversos em pacientes internados em UTI. Revista Brasileira de Enfermagem, 67, 692-699.

Pereira, M. D., de Oliveira, L. C., Costa, C. F. T., de Oliveira Bezerra, C. M., Pereira, M. D., dos Santos, C. K. A., \& Dantas, E. H. M. (2020). A pandemia de COVID-19, o isolamento social, consequências na saúde mental e estratégias de enfrentamento: uma revisão integrativa. Research, Society and Development, 9(7), e652974548-e652974548.

Ramírez-Ortiz, J., Castro-Quintero, D., Lerma-Córdoba, C., Yela-Ceballos, F., \& Escobar Córdoba, F. (2020). Consecuencias de la pandemia COVID-19 en la Salud Mental asociadas al aislamiento social. SciELO Preprints, 1, 1-21. doi: https://doi.org/10.1590/SCIELOPREPRINTS.303

Stegenga, J., Bell, E., \& Matlow, A. (2002). The role of nurse understaffing in nosocomial viral gastrointestinal infections on a general pediatrics ward. Infection Control \& Hospital Epidemiology, 23(3), 133-136.

Stroebe, M., Schut, H., \& Stroebe, W. (2007). Health outcomes of bereavement. The Lancet, 370(9603), 1960-1973. https://doi.org/10.1016/S01406736(07)61816-9

de Vargas, D., \& Braga, A. L. (2006). O enfermeiro de unidade de tratamento intensivo: refletindo sobre seu papel.

Wang, C., Pan, R., Wan, X., Tan, Y., Xu, L., Ho, C. S., \& Ho, R. C. (2020). Immediate psychological responses and associated factors during the initial stage of the 2019 coronavirus disease (COVID-19) epidemic among the general population in china. International Journal of Environmental Research and Public Health, 17(5), 1729. https://doi.org/10.3390/ijerph17051729

Xiang YT, Jin Y, Cheung T. (2020), Joint International Collaboration to Combat Mental Health Challenges During the Coronavirus Disease 2019 Pandemic. JAMA Psychiatry. PubMed PMID: 32275289. Epub 2020/04/11. Eng

Zhang, C., Yang, L., Liu, S., Ma, S., Wang, Y., Cai, Z., \& Zhang, B. (in press), (2020), Survey of insomnia and related social psychological factors among medicais staffs involved with the 2019 novel coronavirus disease outbreak. Frontiers in Psychiatry. https://www.frontiersin.org/articles/10.3389/fpsyt.2020.00306/ 